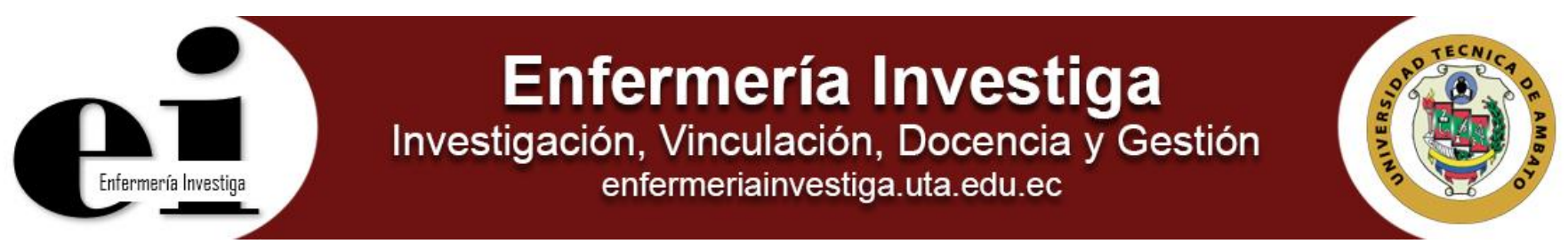

Artículo original

\title{
Gestión del talento humano y su incidencia en la organización de seguridad y salud en el trabajo
}

Management of human talent and its impact on the organization of safety and health at work

\author{
Mauro Darío Albarracín Álvarez¹, Fabiola Beatriz Chasillacta Amores², Verónica del Pilar Gavilanes Fray², Margarita \\ Genoveva Sánchez Yánez², Martha Lucía Guallichico Maura²
}

\footnotetext{
${ }^{1}$ Universidad Técnica de Cotopaxi - Facultad de Ciencias de la Ingeniería y Aplicadas - Carrera de Ingeniería Electromecánica - Latacunga - Ecuador

${ }^{2}$ Universidad Técnica de Ambato - Facultad de Ciencias de la Salud - Carrera de Enfermería - Ambato - Ecuador.
}

Albarracín AMD, Chasillacta AFB. Gestión del talento humano y su incidencia en la organización de seguridad y salud en el trabajo. Enferm Inv (Ambato). 2017; 2(3):100-103.

2477-9172 / 2550-6692 Derechos Reservados ( 2017 Universidad Técnica de Ambato, Carrera de Enfermería. Este es un artículo de acceso abierto distribuido bajo los términos de la Licencia Creative Commons, que permite uso ilimitado, distribución y reproducción en cualquier medio, siempre que la obra original es debidamente citada.

\section{Historia:}

Recibido: 16 julio 2017

Revisado: 20 julio 2017

Aceptado: 09 septiembre 2017

Palabras Claves: Gestión en salud; salud laboral; riesgos laborales

Keywords: Health management; occupational health; occupational risks \begin{abstract}
Resumen
Introducción: Disponer de un Sistema de Seguridad y Salud Ocupacional es una ventaja competitiva, pues se protege al talento humano, los bienes de la organización, los procesos y los ambientes de trabajo.

Objetivo: Determinar la incidencia de la gestión del talento humano en la organización de seguridad y salud en el trabajo del Gobierno Autónomo Descentralizado de la Provincia de Cotopaxi.

Métodos: Se desarrolló un estudio observacional analítico transversal. La recolección de datos se realizó mediante un cuestionario y lista de chequeo para la observación en campo. El universo de estudio estuvo conformado por 366 personas y bajo un muestreo de tipo aleatorio, se obtuvo una muestra de 128 empleados y trabajadores.

Resultados: Se evidenció que el $59.4 \%$, entre empleados y trabajadores desconocieron los factores de riesgo a los que están expuestos en su lugar de trabajo y el $71.1 \%$ desconocieron las medidas preventivas que permitan reducir los mismos. Se reflejó la falta de procesos rigurosos de selección en el marco de la seguridad y salud, así como limitados procesos de inducción, comunicación, información, capacitación y adiestramiento.

Conclusiones: Sería útil la implementación de un manual de funciones con profesiogramas que permitan desarrollar procesos de selección basado en competencias con relación a los factores de riesgos ocupacional por puesto de trabajo.
\end{abstract}

\begin{abstract}
Introduction: The Nursing Career responds to the needs in the health environment identified in the central area of the country. It is duly planned according to the scientific and technical instances of the moment.

Objetive: To diagnose the current situation of the Nursing Career of the UTA according to the needs of the environment in which it is developed and the social order of the same.

Methods: An observational, descriptive and retrospective study was performed. The study period covered from April to September of 2015, the universe of study was composed of 46 teachers who worked in the race in that period and was not selected sample.

Results: The teaching staff only had two auxiliary teachers, only $31.8 \%$ were full-time teachers. $95 \%$ of teachers had affinity with the chairs they teach. The activities of association with the community and management mainly fell to full-time teachers. During the period four books were published and $73 \%$ of the graduates found themselves as public employees. Conclusions: There are no postgraduate programs in order to improve their affinity with the professorships taught by teachers. In the area of research, the creation of multidisciplinary research groups has not been strengthened, while involving undergraduate students, in order to foster a research dynamic since the student starts in his profession.
\end{abstract}

Autor de correspondencia:

Mauro Darío Albarracín Álvarez. Facultad de Ciencias de la Ingeniería y Aplicadas, Universidad Técnica de Cotopaxi, Avenida Simón Rodríguez s/n, Barrio el Ejido, Teléfono: +593 03 2252205, Latacunga, Ecuador. E-mail: mauro.albarracin@utc.edu.ec 


\section{Introducción}

Disponer de un Sistema de Seguridad y Salud Ocupacional es una ventaja competitiva, ya que se protege al talento humano, los bienes de la organización, los procesos y los ambientes de trabajo ${ }^{1-3}$. Garantizar la protección de la seguridad y la salud en el trabajo constituye uno de los elementos esenciales para el aseguramiento de un trabajo digno y éste a su vez contribuye al mejoramiento de la calidad de vida al estar íntimamente relacionados ${ }^{4,5}$. En tal sentido, la Comunidad Andina a través de la "Decisión del Acuerdo de Cartagena 584" decidió adoptar a finales del año 2004 el "Instrumento Andino de Seguridad y Salud en el Trabajo", en el que se establecieron normas fundamentales en esta materia, constituyéndose en la base para la gradual y progresiva armonización de las leyes y los reglamentos que regulen las situaciones particulares de las actividades laborales en cada uno de los países miembros; así como, para impulsar la adopción de directrices sobre sistemas de gestión de la seguridad y la salud en el trabajo.

Por otro lado, el establecimiento de un "Sistema Nacional de Seguridad y Salud en el Trabajo" tuvo como propósito, elevar el nivel de protección de la integridad física y mental de los trabajadores de la región ${ }^{5}$. Con el marco constitucional de la República del Ecuador generado en el año 2008, de manera particular el artículo 326 numeral cinco y en consideración de la legislación vigente, tales como, "Ley de Seguridad Social" y "Código del Trabajo", se estableció como normativa que procura la ejecución del Sistema de Gestión de la Seguridad y Salud en el Trabajo determinado en el "Instrumento Andino de Seguridad y Salud en el Trabajo" más conocido como Resolución 957. Este documento genera directrices generales para el cumplimiento de la normativa técnica y legal en materia de seguridad y salud en el trabajo, por parte de las empresas $u$ organizaciones garantizando que los empleadores provean ambientes saludables y seguros a los trabajadores y que de esa manera coadyuven a la excelencia organizacional ${ }^{6}$.
El cumplimiento de las normas de prevención de riesgos del trabajo en el país es mandatorio en todas organizaciones sujetas al Régimen del Seguro Social, es decir tanto el sector privado como público ${ }^{7,8}$. La inobservancia de estos preceptos en los procesos de selección de los trabajadores, falta de capacitación y adiestramiento según los factores de riesgo por puesto de trabajo y por ende deficiente información y comunicación tanto interna como externa de los factores de riesgo ocupacional, quebranta la seguridad y salud en el trabajo. Por ello reviste importancia gestionar el talento humano y su incidencia en la organización de seguridad y salud en el trabajo, para dar cumplimiento a las exigencias técnico-legales en cada puesto de trabajo ${ }^{9,10}$. El objetivo del presente artículo fue determinar la incidencia de la gestión del talento humano en la organización de seguridad y salud en el trabajo del Gobierno Autónomo Descentralizado (GAD) de la Provincia de Cotopaxi.

\section{Materiales y métodos}

Se desarrolló un estudio observacional analítico transversal. La recolección de datos se realizó mediante un cuestionario y lista de chequeo para la observación en campo, el cuestionario fue aplicado tanto a empleados como a trabajadores de la institución. El universo de estudio estuvo conformado por 366 personas y bajo un muestreo de tipo aleatorio con nivel de confianza del $95 \%$, se obtuvo una muestra de 128 empleados y trabajadores del GAD de la Provincia de Cotopaxi.

La aplicación del cuestionario se realizó al Director de Talento Humano y la lista de chequeo se aplicó al jefe de la Unidad de Seguridad y Salud Ocupacional. La hipótesis planteada fue: La gestión del talento humano incide en la organización de seguridad y salud en el trabajo del GAD de la Provincia de Cotopaxi, para su comprobación se utilizó el método del chi cuadrado.

\section{Resultados}

Tabla 1. Conocimientos sobre medidas de prevención para la reducción de riesgos.

\begin{tabular}{|c|c|c|c|c|c|}
\hline \multirow{2}{*}{\multicolumn{3}{|c|}{$\begin{array}{l}\text { Frecuencias observadas y esperadas: preguntas uno y } \\
\text { cuatro }\end{array}$}} & \multicolumn{2}{|c|}{$\begin{array}{l}\text { 4. ¿Conoce medidas de prevención que } \\
\text { pueden ayudar a reducir los riesgos a los que } \\
\text { está expuesto en su lugar de trabajo? }\end{array}$} & \multirow[t]{2}{*}{ Total } \\
\hline & & & Sí & No & \\
\hline \multirow{6}{*}{$\begin{array}{l}\text { 1. ¿Ha recibido } \\
\text { procesos de } \\
\text { inducción en la } \\
\text { institución? }\end{array}$} & & Recuento & 21 & 25 & 46 \\
\hline & Sí & Frecuencia esperada & 13.3 & 32.7 & 46.0 \\
\hline & & $\%$ del total & $16.4 \%$ & $19.5 \%$ & $35.9 \%$ \\
\hline & & Recuento & 16 & 66 & 82 \\
\hline & No & Frecuencia esperada & 23.7 & 58.3 & 82.0 \\
\hline & & $\%$ del total & $12.5 \%$ & $51.6 \%$ & $64.1 \%$ \\
\hline \multirow{3}{*}{ Total } & & Recuento & 37 & 91 & 128 \\
\hline & & Frecuencia esperada & 37.0 & 91.0 & 128 \\
\hline & & $\%$ del total & $28.9 \%$ & $71.1 \%$ & $100 \%$ \\
\hline
\end{tabular}

Fuente: Encuesta aplicada a empleados y trabajadores del GAD provincia de Cotopaxi.

$x^{2}: 9.80, p=0.05$, valor crítico de chi cuadrado: 3.84 
Tabla 2. Conocimientos sobre exposición a factores de riesgo.

Frecuencias observadas y esperadas: preguntas dos y tres
3. ¿Conoce Ud. los factores de riesgos a los que está expuesto en su lugar de trabajo??

Total

Sí

10

$\begin{array}{ll}5.3 & 7.7\end{array}$

$7.8 \%$

$2.3 \%$

$\%$ del total

Recuento

las funciones que debe cumplir en el desempeño de sus actividades laborales?
Frecuencia esperada $\%$ del total

Recuento No

Frecuencia esperada

$\%$ del total

Recuento

Total

\section{Total}

$x^{2}: 12.59, p=0.05$, valor crítico de chi cuadrado: 5.99

Tabla 3. Nivel de cumplimiento de requisitos técnicolegales.

\begin{tabular}{lcc}
\hline $\begin{array}{l}\text { Nivel de } \\
\text { cumplimiento }\end{array}$ & $\begin{array}{c}\text { Puntaje de } \\
\text { evaluación }\end{array}$ & $\begin{array}{c}\text { Porcentaje } \\
\text { equivalente }\end{array}$ \\
\hline Valor deseado & 16 & $100 \%$ \\
Valor verificado & 0.709 & $4.4 \%$ \\
\hline
\end{tabular}

Fuente: Lista de chequeo aplicada.

\section{Discusión}

Solo el $45.4 \%$ de empleados y trabajadores conocieron las funciones que deben desempeñar en sus lugares de trabajo pues se les ha notificado en forma verbal o escrita, por lo que la mayoría de los encuestados evidenció un déficit de conocimiento de los riesgos a los que está expuesto. Los trabajadores que han sido notificados de manera verbal o escrita sobre las funciones y conocen los riesgos a los que están expuestos fue solo la cuarta parte de la muestra, es decir que el conocimiento tanto de las funciones, así como de los riesgos a los que están expuestos es mínimo ${ }^{11}$.

El elemento correspondiente a la gestión del talento humano dentro del Sistema de Gestión de Seguridad y Salud Ocupacional tuvo un nivel mínimo de cumplimiento correspondiendo al 4.4\%, por lo que, relacionándolo con la información proporcionada en la entrevista, se determinó una gestión del talento humano deficiente ${ }^{11,12}$. Contrastando con los datos obtenidos en la tabulación de la encuesta, se justificó el desconocimiento que existe en cuanto a los factores de riesgos a los que están expuestos los trabajadores por una deficiente gestión.

En referencia a la comprobación de la hipótesis mediante las preguntas uno y cuatro, el valor calculado de chicuadrado con un grado de libertad y $p=0.05$ fue de 9.80 y el valor de tabla fue de 3.84 , es decir que: $x^{2}{ }_{t}=3.84<$ $x^{2}{ }_{\text {calculado }}=9.80$, por lo tanto, la hipótesis es aceptada.

De igual manera en cuanto a las peguntas dos y tres el valor calculado de chi-cuadrado con dos grados de libertad y $p=$ 0.05 fue de 12.59, y el valor de la tabla es de 5.99, es decir que: $x_{t}^{2}=5.99<x^{2}$ calculado $=12.59$, por lo tanto, se rechazó la hipótesis.

\section{Conclusiones}

El déficit de conocimiento de los riesgos laborales a los que están expuestos tanto empleados como trabajadores en sus puestos de trabajo y medidas de prevención se presentó debido a la falta de procesos rigurosos de selección en el marco de la seguridad y salud ocupacional. En este contexto surge la necesidad implementar un manual de funciones con profesiogramas que permitan desarrollar procesos de selección basado en competencias con relación a los factores de riesgos ocupacional por puesto de trabajo. Deben potenciarse procedimientos para la selección, inducción, información, comunicación, capacitación y adiestramiento; que permitan fortalecer y desarrollar la gestión de seguridad y salud ocupacional.

\section{Conflicto de intereses}

Ninguno declarado por los autores.

\section{Referencias}

1. Alles M. Conciliar vida profesional y personal: dos miradas: organizacional e individual [en línea]. Ediciones Granica; 2010. Disponible

https://books.google.es/books?hl=es\&lr=\&id=_F4qBgAAQBAJ\&oi=fn $\mathrm{d} \& \mathrm{pg}=\mathrm{PA} \& \mathrm{dq}=$ Conciliar+vida+profesional+y+personal+Dos+mirada $\mathrm{s}$ :+organizacional+e+individual $+\&$ ots $=\mathrm{mSTdU} 5 \mathrm{dEZs} \& \mathrm{sig}=0-$ 4WLNCgGWRYON2AwVgL8elfQmw\#v=onepage\&q=Conciliar\%20vi da\%20profesional\%20y\%20personal\%20Dos\%20miradas\%3A\%20or ganizacional $\% 20 \mathrm{e} \% 20$ individual $\& \mathrm{f}=\mathrm{false}$

2. Atehortúa F, Bustamante R, Valencia J. Sistema de gestión integral. Una sola gestión, un solo equipo. Medellín: Universidad de Antioquia. 2008.

3. José CA, Aponte JC. Administración de personal: un enfoque hacia la calidad [en línea]. Ecoe Ediciones; 2006. Disponible en: https://books.google.es/books?hl=es\&lr=\&id=1aXmDqJpEc8C\&oi=fn $\mathrm{d} \& \mathrm{pg}=\mathrm{PR} 3 \& \mathrm{dq}=\mathrm{Administraci} \% \mathrm{C} 3 \% \mathrm{~B} 3 \mathrm{n}+\mathrm{de}+$ personal+Un+enfoque+ hacia+la+calidad\&ots=fic9uxVMqJ\&sig=sd0ymRpQ8QNdUYO_OFUV MugxHNc\#v=onepage\&q=Administraci\%C3\%B3n\%20de\%20persona $1 \% 20$ Un\%20enfoque\%20hacia\%20la\%20calidad\&f=false

4. Becerra Rodríguez F, Álvarez Giraldo CM. El talento humano y la innovación empresarial en el contexto de las redes empresariales: el clúster de prendas de vestir en Caldas-Colombia. Estudios gerenciales [en línea]. 2011 [citado 20 de junio de 2017];27(119). Disponible en: http://www.redalyc.org/html/212/21220043010/

5. Hurtado FAA, Vélez REB, de los Ríos JAV. Sistema de gestión integral. Una sola gestión, un solo equipo [en línea]. Universidad de Antioquia; 2008.2 Disponible en: https://books.google.es/books?hl=es\& $\mid \mathrm{r}=\& \mathrm{id}=15 \mathrm{nVyh} 1 \mathrm{Fn} 6 \mathrm{MC} \&$ oi=fnd \&pg=PR13\&dq=Gesti\%C3\%B3n+y+auditoria+de+la+calidad+para+or ganizaciones+publicas+Medellin+hurtado\&ots=ytA6Zrqz8w\&sig=7bZ zaJqlif5Am55QB2Bwy0Sjz-

$\mathrm{Y} \# \mathrm{v}=$ onepage\&q=Gesti\%C3\%B3n\%20y\%20auditoria\%20de\%20la\% 20 calidad\%20para\%20organizaciones $\% 20$ publicas $\% 20$ Medellin $\% 20$ hurtado\& $\mathrm{f}=$ false

6. Torres JL, Jaramillo O. Diseño y análisis del puesto de trabajo: Herramienta para la gestión del talento humano [en línea]. Universidad 
del Norte; $2000 . \quad$ Disponible https://books.google.es/books?hl=es\&lr=\&id=Gr6QCgAAQBAJ\&oi=fn $\mathrm{d} \& p g=P P 1 \& d q=D I S E \% C 3 \% 910+Y+A N \% C 3 \% 81$ LISIS+DEL+PUEST O+DE+TRABAJO+HERRAMIENTAS+PARA+LA+GESTION+DEL+T ALENTO+HUMANO+Barranquilla\&ots=7BTTQUsDfU\&sig=K6cjZD5U XIJ9UDdrKqj3EI3zq-

Y\#v=onepage\&q=DISE\%C3\%910\%20Y\%20AN\%C3\%81LISIS\%20D EL\%2OPUESTO\%20DE\%20TRABAJO\%20HERRAMIENTAS\%20PA RA\%20LA\%20GESTION\%20DEL\%20TALENTO\%20HUMANO\%20B arranquilla\& $\mathrm{f}=\mathrm{false}$

7. Amparo Lozada M, Sánchez AIM. Experiencias de investigación en salud y seguridad en el trabajo [en línea]. Univ. Nacional de Colombia; 2012. Disponible

en:

https://books.google.es/books?hl=es\&lr=\&id=EbgnpLs cuMC\&oi=fnd \&pg=PA7\&dq=EXPERIENCIAS+DE+INVESTIGACI\%C3\%93N+EN+ SALUD+Y+SEGURIDAD+EN+EL+TRABAJO\&ots=j8aHFnomuO\&sig $=\mathrm{L} 2 \mathrm{Kd} 22 \mathrm{Bc3aXykWGN} 6 \mathrm{UwG} 8 \mathrm{Adyew} \# \mathrm{v}=$ onepage $\& \mathrm{q}=\mathrm{EXPERIENCI}$ AS\%20DE\%20INVESTIGACI\%C3\%93N\%20EN\%20SALUD\%20Y\%2 OSEGURIDAD\%20EN\%20EL\%20TRABAJO\&f=false

8. Pareja IST. Propuesta de implementación de un sistema de gestión de seguridad y salud ocupacional bajo la norma OHSAS 18001 en una empresa de capacitación técnica para la industria [en línea]. Pontificia Universidad Católica del Perú. Facultad de Ciencias e Ingeniería. Mención: Ingeniería Industrial; 2012. Disponible en: https://s3.amazonaws.com/academia.edu.documents/36587594/TER
AN_PAREJA ITALA GESTION SEGURIDAD 1.pdf?AWSAccessKe yld=AKIAIWOWYYGZ2Y53UL3A\&Expires $=1505877826 \&$ Signature $=$ \%2Bq\%2FvkWB25\%2B0tVDuYm7E5INkEVjA\%3D\&responsecontent-

disposition=inline\%3B\%20filename\%3DPONTIFICIA UNIVERSIDAD _CATOLICA_DEL_PERU.pdf

9. Robledo FH. Seguridad y salud en el trabajo: Conceptos básicos [en línea]. Ecoe Ediciones; 2016. Disponible en: https://books.google.es/books?hl=es\&lr=\&id=ZKIwDgAAQBAJ\&oi=fn $\mathrm{d} \& \mathrm{pg}=\mathrm{PT} 10 \& \mathrm{dq}=$ Seguridad $+\mathrm{y}+$ salud+en+el+trabajo+Conceptos $+\mathrm{b} \%$ $\mathrm{C} 3 \% \mathrm{~A} 1$ sicos+Bogota\&ots=mPELAhrUir\&sig=KBcddr5BrsJQY0 ZF31j0nFAHUc\#v=onepage\&q\&f=false

10. Idalberto C. Gestión del talento humano. McGraw-Hill; 2002.

11. Saracho JM. Un modelo general de gestión por competencias. Santiago de Chile: RIL. 2005;269.

12. Lablanca C. Apoyo administrativo a la gestión de recursos humanos [en línea]. Ministerio de Educación, Cultura y Deporte; 2015. Disponible https://books.google.es/books?hl=es\&lr=\&id=L07kBgAAQBAJ\&oi=fnd $\& p g=P T 12 \& d q=A p o y o+a d m i n i s t r a t i v o+a+l a+g e s t i \% C 3 \% B 3 n+d e+R e c$ ursos+Humanos\&ots=zkKnpUW7XG\&sig=f2ITUvBeCkf_BVj1iUdM5g RHWEU\#v=onepage\&q=Apoyo\%20administrativo\%20a\%20la\%20ge sti\%C3\%B3n\%20de\%20Recursos\%20Humanos\&f=false 